Andreas Fischer ${ }^{a}$, Matthias Bossard ${ }^{a}$, Stefanie Aeschbacher, Peter Egli, Carolin Cordewener, Joel Estis, John Todd, Martin Risch, Lorenz Risch and David Conen*

\title{
Plasma levels of endothelin-1 and renal function among young and healthy adults
}

DOI 10.1515/cclm-2016-0920

Received October 12, 2016; accepted January 10, 2017; previously published online February 21, 2017

\section{Abstract}

Background: Endothelin-1 (ET-1), a vasoconstrictive and pro-inflammatory peptide, is associated with several cardiovascular risk factors and outcomes. We aimed to investigate the association of plasma ET-1 levels and renal function among young and healthy adults.

Methods: Individuals aged 25-41 years were enrolled in a population-based cohort study. Main exclusion criteria were established kidney disease, cardiovascular diseases, diabetes mellitus and a body mass index $>35 \mathrm{~kg} / \mathrm{m}^{2}$.

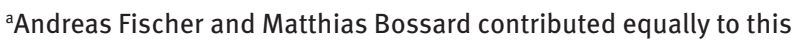
work and are shared first authors.

*Corresponding author: Assoc. Prof. Dr. David Conen, MD, MPH, Population Health Research Institute, David Braley Cardiac, Vascular and Stroke Research Institute, McMaster University, 237 Barton Street East, Hamilton, ON L8L 2X2, Canada, Phone: + 1905905522 (Ext. 1155), E-mail: conend@mcmaster.ca; Cardiology Division, Department of Medicine, University Hospital Basel, Basel, Switzerland; and Cardiovascular Research Institute Basel, University Hospital Basel, Basel, Switzerland

Andreas Fischer, Stefanie Aeschbacher and Peter Egli: Cardiology Division, Department of Medicine, University Hospital Basel, Basel, Switzerland; and Cardiovascular Research Institute Basel, University Hospital Basel, Basel, Switzerland

Matthias Bossard: Cardiology Division, Department of Medicine, University Hospital Basel, Basel, Switzerland; Cardiovascular Research Institute Basel, University Hospital Basel, Basel, Switzerland; Division of Cardiology, Hamilton General Hospital, Hamilton Health Sciences, Hamilton, ON, Canada; and Population Health Research Institute, David Braley Cardiac, Vascular and Stroke Research Institute, Hamilton, ON, Canada

Carolin Cordewener: Cardiovascular Research Institute Basel, University Hospital Basel, Basel, Switzerland

Joel Estis and John Todd: Singulex, Inc., Clinical Research, Alameda, CA, USA

Martin Risch: Labormedizinisches Zentrum Dr. Risch, Schaan, Principality of Liechtenstein; and Division of Laboratory Medicine, Kantonsspital Graubünden, Chur, Switzerland

Lorenz Risch: Labormedizinisches Zentrum Dr. Risch, Schaan, Principality of Liechtenstein; Division of Clinical Biochemistry, Medical University, Innsbruck, Austria; and Private University, Triesen, Principality of Liechtenstein
Fasting venous plasma samples were used to measure creatinine, cystatin C and ET-1. The estimated glomerular filtration rate (eGFR) was calculated using the creatinine based chronic kidney disease epidemiology collaboration (CKD-EPI) formula. Multivariable regression models were constructed to assess interrelationships of plasma ET-1 with parameters of renal function.

Results: Median age of the 2139 participants was 37 years, 47\% males. Median creatinine and eGFR were $67 \mu \mathrm{mol} / \mathrm{L}$ and $112 \mathrm{~mL} / \mathrm{min} / 1.73 \mathrm{~m}^{2}$, respectively. Using quartile one as the reference group, the $\beta$-coefficients ( $95 \%$ confidence intervals [CIs]) for eGFR were 0.06 ( -1.22 to 1.35), -0.66 ( -1.95 to 0.62$)$ and -1.70 ( -3.01 to -0.39$)$ for quartiles $2-4$ ( $p$-for-trend $=0.0056$ ), respectively and $\beta$-coefficients (95\% CIs) for cystatin C were 0.002 ( -0.01 to 0.02 ), 0.02 (0.003-0.03) and $0.03(0.01-0.04)$ for quartiles $2-4$ (p-fortrend $<0.0001)$, respectively. Using ET-1 as a continuous variable, the $\beta$-coefficient (95\% CI) for eGFR per 1-unit increase was $-1.82(-3.19$ to $-0.44, \mathrm{p}=0.0095)$ and 0.02 (0.01-0.04, $\mathrm{p}=0.0003)$ for cystatin C. Similar results were found between creatinine and ET-1 levels.

Conclusions: ET-1 levels are strongly associated with parameters of renal function among young and healthy adults, suggesting an important role of ET-1 and endothelial function in the regulation of kidney function.

Keywords: chronic kidney disease; endothelin-1; epidemiology; glomerular filtration rate; population based; renal function.

\section{Introduction}

Chronic kidney disease (CKD) represents a growing public health burden, which comes along with aging societies and rising prevalence of cardiovascular diseases and diabetes [1-4]. CKD is a major cause of morbidity and mortality [4-6]. Consequently, early detection and prevention of CKD is of major importance [7].

Endothelin-1 (ET-1) is a 21-amino-acid, mainly endothelium-derived, peptide with vasoconstrictive, inflammatory and mitogenic properties [8-11]. Elevated ET-1 levels have been associated with hypertension, endothelial dysfunction and atherosclerotic diseases [9, 
12-17]. Mostly experimental studies have also indicated that ET-1 is involved in regulation of renal function, but population-based studies on this issue are sparse [18-22].

Historically, the determination of plasma ET-1 levels has been time-consuming and cost-intensive. However, the advent of simple high-sensitivity assays lately enabled rapid determination plasma-derived ET-1 [23, 24]. These novel assays simplified the assessment of ET-1 levels and enable its determination in large population-based studies. In order to gain potential further insights into the role of the endothelin system in the regulation of kidney function, we evaluated the relationships of ET-1 with kidney parameters among young and healthy individuals without established kidney diseases.

\section{Materials and methods}

\section{Study participants}

The "Genetic and phenotypic determinants of blood pressure and other cardiovascular risk factors" (GAPP) study is an ongoing prospective population-based cohort study among young and healthy adults in the Principality of Liechtenstein. The detailed study design has been previously published [25]. In brief, all inhabitants of the Principality of Liechtenstein aged between 25 and 41 years were invited to participate in this study. Our main exclusion criteria were established kidney disease, known cardiovascular disease or diabetes mellitus, a body mass index (BMI) $>35 \mathrm{~kg} / \mathrm{m}^{2}$ or any other severe illness. Each participant gave written informed consent and the Local Ethical Commission approved the study protocol. This study has been conducted by following the tenets of the Declaration of Helsinki.

Between 2010 and 2013, we enrolled 2170 individuals. For the purpose of this analysis, we excluded 30 individuals with missing information on either plasma ET-1, plasma creatinine, urinary albumin, blood pressure (BP) or physical activity. We also excluded one participant with a plasma creatinine level of $331.5 \mu \mathrm{mol} / \mathrm{L}$. Thus, we included 2139 individuals in the present study.

\section{Blood and urine sampling}

Fasting venous blood and morning urinary samples were collected from every participant. After centrifugation, the samples were immediately stored at $-80^{\circ} \mathrm{C}$. ET-1 was obtained from frozen EDTA plasma samples by using a high-sensitivity, single-molecule counting assay (Erenna ${ }^{\circledR}$ Immunoassay System, Singulex, Inc., Alameda, CA, USA) [24]. OurET-1 assay's limits ofblankand quantification were $0.07 \mathrm{pg} / \mathrm{mL}$ and $0.33 \mathrm{pg} / \mathrm{mL}$, respectively. Inter-assay coefficients of variation were $7 \%$ at an ET-1 concentration of $1.2 \mathrm{pg} / \mathrm{mL}$ and $6 \%$ at an ET-1 concentration of $1.8 \mathrm{pg} / \mathrm{mL}$ [24].

Plasma levels of creatinine, cystatin $\mathrm{C}$, N-terminal pro brainnatriuretic peptide (NT-pro-BNP), high-sensitivity $\mathrm{C}$ reactive protein (hs-CRP), low-density lipoprotein cholesterol (LDL-C), as well as urinary levels of creatinine and albumin were determined in fresh samples using a Roche Cobas 6000 analyzer (F. Hoffmann-La Roche Ltd., Switzerland). Urinary sodium, creatinine and albumin levels were also measured using a Roche Cobas 6000 analyzer. Glycated hemoglobin $\left(\mathrm{HbA}_{1 \mathrm{c}}\right)$ was analyzed using high performance liquid chromatography (Bio-Rad D-10, Bio-Rad Laboratories AG, Switzerland) [25]. We calculated the estimated 24-h albuminuria and a urinary albumin to creatinine ratio (UACR) of at least $30 \mathrm{mg} / 24 \mathrm{~h}$ was defined as an albuminuria, as previously described [26]. The glomerular filtration rate (eGFR) was estimated using the chronic kidney disease epidemiology collaboration (CKD-EPI) formula based on plasma creatinine [27]. For the estimation of the $24 \mathrm{~h}$ urinary sodium excretion, we used morning spot urine samples and applied Kawasaki's formula [28]. Prediabetes/diabetes was defined as a $\mathrm{HbA}_{1 \mathrm{c}}$ level > 5.7\% [29].

\section{Other study variables}

Information about personal, medical, lifestyle and nutritional factors was collected using standardized questionnaires. The highest education level achieved was categorized into 'high school', 'college' or 'university' degree. Self-assessed smoking status was classified as current, former or never smoking. We dichotomized drinking habits as drinkers versus non-drinkers. The individual physical activity questionnaire (IPAQ) was applied for the evaluation of the subjects' physical activity level [25].

We obtained conventional BP measurements after at least $5 \mathrm{~min}$ of rest using a validated oscillometric device (Microlife BP3AG1, Microlife AG, Switzerland). The mean of the second and third measurement was used for BP-related analyses. Hypertension was defined as a systolic $\mathrm{BP} \geq 140 \mathrm{mmHg}$, a diastolic $\mathrm{BP} \geq 90 \mathrm{mmHg}$ or the intake of antihypertensive drugs. Body weight and height were measured in a standardized manner [25]. BMI was calculated by dividing weight in kilograms by height in meters squared. Bioelectrical impedance was used to estimate the body composition of all subjects using an established methodology and a validated device (BIA ego fit, 2010, Germany) [25].

\section{Statistical analyses}

We stratified the baseline characteristics by quartiles of ET-1. The distribution pattern of continuous variables was assessed using kurtosis, skewness and visual inspection of the histograms. The baseline characteristics of continuous variables were presented as medians (interquartile range) and compared using ANOVA or Kruskal-Wallis tests as appropriate. Categorical variables were compared using $\chi^{2}$-tests.

We used multivariable linear regression models to compare the $\beta$-coefficients of creatinine, cystatin $\mathrm{C}$ and the eGFR across quartiles of ET-1 and to adjust for potential confounders. All models were adjusted for a predefined set of covariates, including age, sex, BMI, LDL-C, $\mathrm{HbA}_{1 \mathrm{c}}$, systolic BP, hs-CRP, estimated urinary sodium excretion, estimated body water and muscle mass, physical activity, smoking status, alcohol consumption and education level. Since all associations were approximately linear, additional analyses were performed using ET-1 as a log-transformed continuous variable as the predictor of interest. Additionally, a logistic regression model was 
constructed to assess the relationship between ET-1 levels and UACR, using the same variables indicated above.

Stratified regression models were applied to assess the relationships between ET-1 and eGFR across pre-specified subgroups, including age, sex, BMI, prediabetes, hypertension and smoking, and formal differences were assessed using multiplicative interaction terms in the non-stratified models. Categorical variables were introduced in all multivariable models using binary indicator variables. All statistical analyses were performed with SAS version 9.4 (SAS Institute, Inc., Cary, NC, USA). A p-value of $<0.05$ was pre-specified to indicate statistical significance.

\section{Results}

Baseline demographics of the 2139 subjects according to quartiles of ET-1 are displayed in Table 1. Quartile-specific median ET-1 levels were 1.86, 2.33, 2.76, and 3.49 pg/mL.
The median (interquartile range) eGFR was 112 (103-118) $\mathrm{mL} / \mathrm{min} / 1.73 \mathrm{~m}^{2}$. Being in a higher ET-1 quartile was significantly associated with male sex, and higher levels of BP, BMI, LDL-C, hs-CRP and estimated urinary sodium excretion. Current smokers and individuals with higher physical activity levels had also higher ET-1 levels. There was a significant linear increase across ET-1 quartiles for creatinine, cystatin $\mathrm{C}$ and eGFR $(\mathrm{p}<0.0001, \mathrm{p}<0.0001$ and $\mathrm{p}=0.0043$, respectively). We found nonlinear differences across ET-1 quartiles for body water $(p=0.011)$, alcohol intake $(\mathrm{p}=0.006)$ and urinary albumin-to-creatinine ratio $(p=0.022)$.

Regression analyses for the association of creatinine, cystatin $\mathrm{C}$, the eGFR and albuminuria with plasma ET-1 levels are displayed in Table 2. The significant relationships of ET-1 with creatinine, cystatin C and eGFR on univariable assessment persisted after adjustment for

Table 1: Baseline characteristics according to quartiles of endothelin-1.

\begin{tabular}{|c|c|c|c|c|c|}
\hline $\begin{array}{l}\text { Total }(n=2139) \\
\text { ET-1 range, } \mathrm{pg} / \mathrm{mL}\end{array}$ & $\begin{array}{r}\text { Quartile } 1(n=537) \\
0.11-2.12\end{array}$ & $\begin{array}{r}\text { Quartile } 2(n=535) \\
2.12-2.54\end{array}$ & $\begin{array}{r}\text { Quartile } 3(n=536) \\
2.54-3.06\end{array}$ & $\begin{aligned} \text { Quartile } 4(n & =531) \\
& \geq 3.06\end{aligned}$ & p-Value \\
\hline Age, years & $35.9(30.6-39.9)$ & $37.0(31.4-40.5)$ & $36.3(31.1-40.2)$ & $37.3(31.7-40.4)$ & 0.076 \\
\hline Sex, male (\%) & $204(38.0)$ & $236(44.1)$ & $267(49.8)$ & $289(54.4)$ & $<0.0001$ \\
\hline Highest education level (\%) & & & & & 0.51 \\
\hline High school & $55(10.3)$ & $41(7.7)$ & $41(7.7)$ & $38(7.2)$ & \\
\hline College & $276(51.9)$ & $293(55.3)$ & $285(53.6)$ & $295(56.2)$ & \\
\hline University degree & $201(37.8)$ & $196(37.0)$ & $206(38.7)$ & $192(36.6)$ & \\
\hline Smoking (\%) & & & & & 0.0006 \\
\hline Current & 109 (20.3) & $89(16.6)$ & $122(22.8)$ & $150(28.3)$ & \\
\hline Past & $121(22.5)$ & $132(24.7)$ & $124(23.1)$ & $122(23.0)$ & \\
\hline Never & $307(57.2)$ & $314(58.7)$ & $290(54.1)$ & $258(48.7)$ & \\
\hline Physical activity, minutes & $120(60-300)$ & $180(60-360)$ & $180(60-360)$ & $180(60-420)$ & 0.039 \\
\hline Alcohol intake, g/day & $0.0(0.0-1.4)$ & $0.0(0.0-1.7)$ & $0.6(0.0-2.0)$ & $0.6(0.0-1.7)$ & 0.006 \\
\hline $\mathrm{BMI}, \mathrm{kg} / \mathrm{m}^{2}$ & $23.9(21.7-26.7)$ & $23.9(21.5-27.0)$ & $24.0(21.8-26.6)$ & $24.7(22.1-27.9)$ & 0.008 \\
\hline Muscle mass, $\%$ & $34.8(32.3-37.8)$ & $35.1(32.7-37.9)$ & $35.6(32.8-38.5)$ & $35.1(32.4-38.0)$ & 0.14 \\
\hline Body water, \% & $54.0(50.2-57.3)$ & $54.5(51.0-58.4)$ & $55.3(51.5-58.6)$ & $54.3(50.8-58.3)$ & 0.011 \\
\hline Systolic BP, mmHg & $118(110-123)$ & $120(111-128)$ & $120(111-129)$ & $122(113-131)$ & $<0.0001$ \\
\hline Diastolic BP, mmHg & $77(72-83)$ & $78(72-84)$ & $79(72-84)$ & $79(73-86)$ & 0.0017 \\
\hline Antihypertensive TRT (\%) & $8(1.5)$ & $11(2.1)$ & $6(1.1)$ & $10(1.9)$ & 0.63 \\
\hline NT-proBNP, pg/mL & $36(16-60)$ & $36(20-58)$ & $31(18-58)$ & $34(18-60)$ & 0.46 \\
\hline Hs-CRP, mg/L & $0.9(0.5-1.8)$ & $0.9(0.5-1.7)$ & $0.9(0.5-1.8)$ & $1.1(0.5-2.3)$ & 0.002 \\
\hline LDL-C, mmol/L & $2.77(2.28-3.34)$ & $2.90(2.33-3.50)$ & $2.93(2.39-3.50)$ & $2.95(2.41-3.63)$ & 0.0021 \\
\hline Hemoglobin $A_{1 c}, \%$ & $5.4(5.1-5.6)$ & $5.4(5.2-5.7)$ & $5.4(5.1-5.6)$ & $5.4(5.2-5.7)$ & 0.037 \\
\hline Prediabetes (\%) & 107 (19.9) & $139(26.0)$ & $123(23.0)$ & $139(26.2)$ & 0.052 \\
\hline Creatinine, $\mu \mathrm{mol} / \mathrm{L}$ & $63.6(55.7-74.3)$ & $66.3(57.4-75.1)$ & $68.1(57.5-77.8)$ & $69.0(60.1-79.6)$ & $<0.0001$ \\
\hline Cystatin C, mg/L & $0.77(0.70-0.85)$ & $0.77(0.69-0.86)$ & $0.79(0.71-0.87)$ & $0.80(0.73-0.88)$ & $<0.0001$ \\
\hline $\mathrm{eGFR}, \mathrm{mL} / \mathrm{min} / 1.73 \mathrm{~m}^{2 \mathrm{a}}$ & $114(105-119)$ & $112(106-118)$ & $112(103-118)$ & $110(101-118)$ & 0.0043 \\
\hline UACR, mg/24 h & $2.65(1.54-4.97)$ & $2.97(1.81-5.79)$ & $2.74(1.62-4.94)$ & $2.68(1.58-5.03)$ & 0.022 \\
\hline Urinary sodium excretion, $\mathrm{g} / 24 \mathrm{~h}^{\mathrm{b}}$ & $3.98(3.21-4.84)$ & $3.98(3.17-4.93)$ & $4.06(3.36-5.11)$ & $4.18(3.30-5.22)$ & 0.032 \\
\hline
\end{tabular}

p-Values were based on Kruskal-Wallis tests or $\chi^{2}$-tests, as appropriate. Data are median (interquartile range) or number (percentage). BMI, body mass index; BP, blood pressure; TRT, treatment; NT-proBNP, N-terminal pro-brain natriuretic peptide; LDL-C, low-density lipoprotein cholesterol; Hs-CRP, high-sensitive C-reactive protein; eGFR, estimated glomerular filtration rate; UACR, urine albumin-to-creatinine ratio.

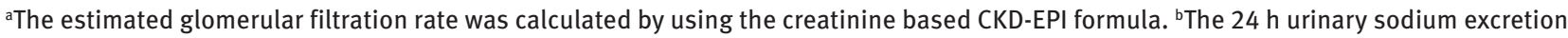
was estimated by applying Kawasaki’s formula. 
multiple potential confounders. Compared to the lowest quartile, the eGFR of individuals in the highest quartile was significantly lower, as shown in Figure 1. On the other hand, we did not find a significant association between ET-1 levels and albuminuria, as shown in Table 2. There were no significant interactions for the association between ET-1 and the eGFR, as shown in Table 3.

\section{Discussion}

This study has several noteworthy findings. First, by applying a novel high-sensitivity assay, we were able to quantify plasma ET-1 levels in a large cohort of young and healthy adults at low cardiovascular risk. Second, our analyses revealed significant relationships of plasma ET-1 with creatinine, cystatin C and eGFR among these individuals without renal or atherosclerotic disease. By including a broad set of important clinical variables to our models, we further reduced the potential for residual confounding.

Earlier studies indicated that the endothelin system, particularly ET-1, directly influence the vasomotor function and thus renal blood flow, which consequently impacts renal function [30,31]. It has also been shown that the endothelin system plays a key role in common pathophysiological processes involved in most renal disorders, and ET-1, for example, directly promotes mesangial cell proliferation and glomerular sclerosis [21, 32]. Finally, smaller studies including patients with established renal disease have also shown that higher plasma concentrations of ET-1 are inversely associated to measured GFR [33].

By highlighting a relationship between plasma ET-1 and renal parameters in individuals with a normal function (the median eGFR in this study was $112 \mathrm{~mL} /$

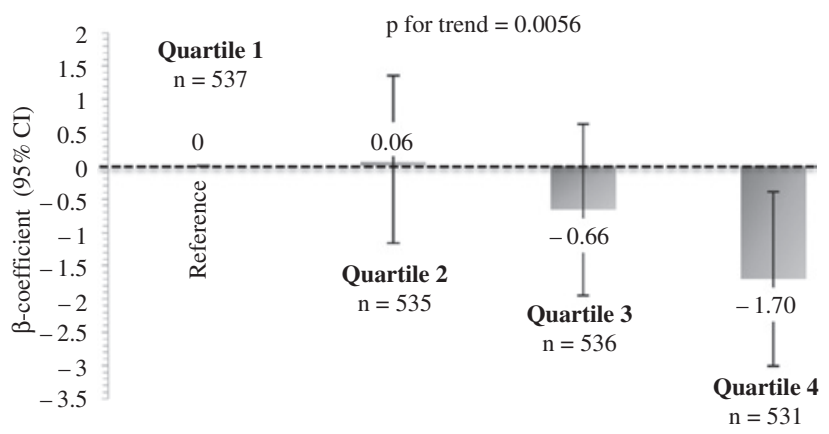

Figure 1: Relationship between plasma endothelin-1 and the estimated glomerular filtration rate.

Data are $\beta$ (95\% confidence intervals) for ET-1 quartiles $2-4$, respectively using quartile 1 as the reference group. 
Table 3: Subgroup analyses for the relationship between plasma endothelin-1 and the estimated glomerular filtration rate (eGFR).

\begin{tabular}{|c|c|c|c|}
\hline & $\mathbf{n}$ & $\beta(95 \% \mathrm{Cl})$ & $p$ for interaction \\
\hline \multicolumn{4}{|l|}{ Sex } \\
\hline Male & 984 & $-3.10(-5.26$ to -0.93$)$ & \multirow[t]{2}{*}{0.39} \\
\hline Female & 1123 & $-1.18(-2.99$ to 0.62$)$ & \\
\hline \multicolumn{4}{|c|}{ Age, years } \\
\hline$\geq 35$ & 1204 & $-2.23(-3.97$ to -0.50$)$ & \multirow[t]{2}{*}{0.81} \\
\hline$<35$ & 899 & $-1.02(-3.40$ to 1.35$)$ & \\
\hline \multicolumn{4}{|c|}{ Prediabetes/diabetes } \\
\hline Yes & 498 & $-3.39(-6.66$ to -0.12$)$ & \multirow[t]{2}{*}{0.37} \\
\hline No & 1605 & $-1.57(-3.10$ to -0.04$)$ & \\
\hline \multicolumn{4}{|c|}{$\mathrm{BMI}, \mathrm{kg} / \mathrm{m}^{2}$} \\
\hline$\geq 25$ & 864 & $-2.06(-4.05$ to -0.07$)$ & \multirow[t]{2}{*}{0.89} \\
\hline$<25$ & 1239 & $-1.61(-3.54$ to 0.33$)$ & \\
\hline \multicolumn{4}{|c|}{ Hypertension } \\
\hline Yes & 288 & $-1.50(-5.11$ to 2.10$)$ & \multirow[t]{2}{*}{0.63} \\
\hline No & 1815 & $-2.12(-3.61$ to -0.62$)$ & \\
\hline \multicolumn{4}{|c|}{ Current smoking } \\
\hline Yes & 466 & $-0.57(-3.39$ to 2.25$)$ & \multirow[t]{2}{*}{0.41} \\
\hline No & 1638 & $-2.03(-3.61$ to -0.45$)$ & \\
\hline
\end{tabular}

The $\beta$-coefficients ( $95 \%$ confidence intervals) represent the increase of the estimated glomerular filtration rate (in $\mathrm{mL} / \mathrm{min} / 1.73 \mathrm{~m}{ }^{2}$ ) per unit of log transformed endothelin-1 levels. All coefficients were adjusted for sex, age, body mass index, systolic blood pressure, low-density lipoprotein cholesterol, prediabetes/diabetes, high-sensitive C-reactive protein, $\mathrm{N}$-terminal pro-brain natriuretic peptide, estimated urinary sodium excretion, estimated muscle mass, estimated total body water, education level, alcohol consumption, physical activity and current smoking.

$\min / 1.73 \mathrm{~m}^{2}$ ), we are confirming and expanding the existing, mostly experimental based knowledge about the endothelin-system to a population-based setting. Furthermore, it is noteworthy that the relationship between ET- 1 and the eGFR remained almost unaffected by adjustment for hs-CRP and NT-pro-BNP, whereas the endothelin system has been shown to trigger inflammation and natriuretic peptides synthesis, which in turn also influence the renal physiology [34, 35]. Hence, this might underscore the independent role of ET-1 in regulating the renal function. Our data actually suggest that ET-1 may be a sensitive marker of kidney blood flow and/or renal function even in adults with high-normal eGFR levels. Future studies should assess whether plasma-derived ET-1 might become a promising biomarker to estimate renal blood flow and function, and to identify individuals at risk for progression of kidney disease.

In this context, one needs to take in account that the prevalence of chronic kidney disease continuously increases with advancing age, affecting more than $20 \%$ of all individuals older than 60 years and $35 \%$ of those older than 70 years $[1,3,4]$. It is now also well-established that even early-stage renal failure adversely impacts general health and represents an independent cardiovascular risk factor $[1,3,4]$. Consequently, early recognition of adverse renal changes and ultimately prevention of a chronic renal condition, particularly cost-intensive and deadly end-stage renal disease (ESRD), are of utmost clinical importance.

In contrast to our study, earlier studies showed relationships between ET-1 and albuminuria [33, 36]. However, those studies mostly included either elderly individuals or patients with established renal disease, which may influence the described association there [37]. We presume that the earlier shown association between ET-1 and albuminuria might be more important among individuals with advanced renal damage. Additionally, we only had a sample of 52 patients with albuminuria, which might limit the power to show an association. Moreover, this finding could also mean that the relationship between plasma ET-1 and renal parameters in young and healthy adults predominantly reflects renal perfusion and not tubular or glomerular damage.

Strengths of this population based-study are the large and well-characterized cohort of young and healthy individuals and the availability of measurable ET-1 levels in virtually all participants. We are aware of certain limitations which apply to our study: First, the cross-sectional design of this study does not allow to draw causal inferences; second, our study cohort included low risk and mainly white adults and the generalizability to other populations remains unknown; third, we did neither directly 
measure the renal function nor invasively estimate the endothelial function. However, the eGFR assessment with the CKD-EPI formula reflects a broadly validated tool for determination renal function, even among young individuals [38-40]. In addition, it has been shown that levels of ET-1 are closely and directly linked to endothelial dysfunction and presence of atherosclerosis [24, 41].

\section{Conclusions}

In young and healthy adults, plasma ET-1 levels are significantly associated with parameters of renal function. These findings suggest that ET-1 may play an important role in the regulation of renal physiology, especially regulation of renal blood flow. Future studies should assess the role of ET-1 in a clinical context and clarify whether this biomarker could improve risk stratification and identify individuals at risk for development of chronic renal disease.

Author contributions: All the authors have accepted responsibility for the entire content of this submitted manuscript and approved submission.

Research funding: The Swiss National Science Foundation, the Liechtenstein Government, the Swiss Heart Foundation, the Swiss Society of Hypertension, the University of Basel, the University Hospital Basel, the Hanela Foundation, Schiller AG and Novartis Foundation supported the GAPP study. Endothelin-1 was measured free of charge by Singulex, Inc., Alameda, CA 94502, USA. Samples were provided and tested for endothelin-1 at Singulex in a blinded manner without access to any variables in the study database other than patient identifiers. Matthias Bossard was supported by grants of the University of Basel and Freiwillige Akademische Gesellschaft (FAG) Basel.

Employment or leadership: John Todd and Joel Estis are employees of Singulex, Inc.

Honorarium: None declared.

Competing interests: The funding organization(s) played no role in the study design; in the collection, analysis, and interpretation of data; in the writing of the report; or in the decision to submit the report for publication.

\section{References}

1. Coresh J, Selvin E, Stevens LA, Manzi J, Kusek JW, Eggers P, et al. Prevalence of chronic kidney disease in the United States. J Am Med Assoc 2007;298:2038-47.

2. James MT, Hemmelgarn BR, Tonelli M. Early recognition and prevention of chronic kidney disease. Lancet 2010;375:1296-309.
3. Eckardt KU, Coresh J, Devuyst O, Johnson RJ, Kottgen A, Levey AS, et al. Evolving importance of kidney disease: from subspecialty to global health burden. Lancet 2013;382:158-69.

4. Ene-lordache B, Perico N, Bikbov B, Carminati S, Remuzzi A, Perna A, et al. Chronic kidney disease and cardiovascular risk in six regions of the world (ISN-KDDC): a cross-sectional study. Lancet Glob Health 2016;4:e307-19.

5. Astor BC, Hallan SI, Miller ER, 3rd, Yeung E, Coresh J. Glomerular filtration rate, albuminuria, and risk of cardiovascular and all-cause mortality in the US population. Am J Epidemiol 2008;167:1226-34.

6. Chugh S, Chugh Y. Radial artery occlusion: size and more-how small is too small? J Invasive Cardiol 2015;27:E113.

7. Ruggenenti P, Perticucci E, Cravedi P, Gambara V, Costantini M, Sharma SK, et al. Role of remission clinics in the longitudinal treatment of CKD. J Am Soc Nephrol 2008;19:1213-24.

8. Yanagisawa M, Kurihara H, Kimura S, Tomobe Y, Kobayashi M, Mitsui Y, et al. A novel potent vasoconstrictor peptide produced by vascular endothelial cells. Nature 1988;332:411-5.

9. Lerman A, Edwards BS, Hallett JW, Heublein DM, Sandberg SM, Burnett JC Jr. Circulating and tissue endothelin immunoreactivity in advanced atherosclerosis. N Engl J Med 1991;325:997-1001.

10. Haynes WG, Webb DJ. Contribution of endogenous generation of endothelin-1 to basal vascular tone. Lancet 1994;344:852-4.

11. Speed JS, Pollock DM. Endothelin, kidney disease, and hypertension. Hypertension 2013;61:1142-5.

12. Lerman A, Holmes DR Jr., Bell MR, Garratt KN, Nishimura RA, Burnett JC Jr. Endothelin in coronary endothelial dysfunction and early atherosclerosis in humans. Circulation 1995;92:2426-31.

13. Barton M, Haudenschild CC, d'Uscio LV, Shaw S, Munter K, Luscher TF. Endothelin ETA receptor blockade restores NOmediated endothelial function and inhibits atherosclerosis in apolipoprotein E-deficient mice. Proc Natl Acad Sci USA 1998;95:14367-72.

14. Verma S, Li SH, Badiwala MV, Weisel RD, Fedak PW, Li RK, et al. Endothelin antagonism and interleukin- 6 inhibition attenuate the proatherogenic effects of $\mathrm{C}$-reactive protein. Circulation 2002;105:1890-6.

15. Amiri F, Virdis A, Neves MF, Iglarz M, Seidah NG, Touyz RM, et al. Endothelium-restricted overexpression of human endothelin-1 causes vascular remodeling and endothelial dysfunction. Circulation 2004;110:2233-40.

16. Reriani M, Raichlin E, Prasad A, Mathew V, Pumper GM, Nelson $\mathrm{RE}$, et al. Long-term administration of endothelin receptor antagonist improves coronary endothelial function in patients with early atherosclerosis. Circulation 2010;122:958-66.

17. Li MW, Mian MO, Barhoumi T, Rehman A, Mann K, Paradis P, et al. Endothelin-1 overexpression exacerbates atherosclerosis and induces aortic aneurysms in apolipoprotein E knockout mice. Arterioscler Thromb Vasc Biol 2013;33:2306-15.

18. Orisio S, Benigni A, Bruzzi I, Corna D, Perico N, Zoja C, et al. Renal endothelin gene expression is increased in remnant kidney and correlates with disease progression. Kidney Int 1993;43:354-8.

19. Hocher B, Thone-Reineke C, Rohmeiss P, Schmager F, Slowinski T, Burst V, et al. Endothelin-1 transgenic mice develop glomerulosclerosis, interstitial fibrosis, and renal cysts but not hypertension. J Clin Invest 1997;99:1380-9. 
20. Fligny C, Barton M, Tharaux PL. Endothelin and podocyte injury in chronic kidney disease. Contrib Nephrol 2011;172:120-38.

21. Lenoir O, Milon M, Virsolvy A, Henique C, Schmitt A, Masse JM, et al. Direct action of endothelin-1 on podocytes promotes diabetic glomerulosclerosis. J Am Soc Nephrol 2014;25:1050-62.

22. Barton M, Sorokin A. Endothelin and the glomerulus in chronic kidney disease. Semin Nephrol 2015;35:156-67.

23. Todd J, Freese B, Lu A, Held D, Morey J, Livingston R, et al. Ultrasensitive flow-based immunoassays using single-molecule counting. Clin Chem 2007;53:1990-5.

24. Bossard M, Pumpol K, van der Lely S, Aeschbacher S, Schoen T, Krisai P, et al. Plasma endothelin-1 and cardiovascular risk among young and healthy adults. Atherosclerosis 2015;239:186-91.

25. Conen D, Schon T, Aeschbacher S, Pare G, Frehner W, Risch M, et al. Genetic and phenotypic determinants of blood pressure and other cardiovascular risk factors (GAPP). Swiss Med Wkly 2013;143:w13728.

26. Nathan DM, Rosenbaum C, Protasowicki VD. Single-void urine samples can be used to estimate quantitative microalbuminuria. Diabetes Care 1987;10:414-8.

27. KDIGO 2012 Clinical Practice Guideline for the Evaluation and Management of Chronic Kidney Disease. Kidney Int 2013;3:1-150.

28. Kawasaki T, Itoh K, Uezono K, Sasaki H. A simple method for estimating $24 \mathrm{~h}$ urinary sodium and potassium excretion from second morning voiding urine specimen in adults. Clin Exp Pharmacol Physiol 1993;20:7-14.

29. Standards of medical care in diabetes-2012. Diabetes Care 2012;35(Suppl 1):S11-63.

30. Cody RJ, Ljungman S, Covit AB, Kubo SH, Sealey JE, Pondolfino K, et al. Regulation of glomerular filtration rate in chronic congestive heart failure patients. Kidney Int 1988;34:361-7.

31. Kohan DE, Inscho EW, Wesson D, Pollock DM. Physiology of endothelin and the kidney. Compr Physiol 2011;1:883-919.
32. Kohan DE, Barton M. Endothelin and endothelin antagonists in chronic kidney disease. Kidney Int 2014;86:896-904.

33. Zeravica R, Cabarkapa V, Ilincic B, Sakac V, Mijovic R, Nikolic S, et al. Plasma endothelin-1 level, measured glomerular filtration rate and effective renal plasma flow in diabetic nephropathy. Ren Fail 2015;37:681-6.

34. Magga J, Vuolteenaho O, Marttila M, Ruskoaho H. Endothelin-1 is involved in stretch-induced early activation of B-type natriuretic peptide gene expression in atrial but not in ventricular myocytes: acute effects of mixed ET(A)/ET(B) and AT1 receptor antagonists in vivo and in vitro. Circulation 1997;96:3053-62.

35. Kohan DE. Endothelin, hypertension and chronic kidney disease: new insights. Curr Opin Nephrol Hypertens 2010;19:134-9.

36. Zanatta CM, Gerchman F, Burttet L, Nabinger G, Jacques-Silva MC, Canani LH, et al. Endothelin-1 levels and albuminuria in patients with type 2 diabetes mellitus. Diabetes Res Clin Pract 2008;80:299-304.

37. Seliger SL, Salimi S, Pierre V, Giffuni J, Katzel L, Parsa A. Microvascular endothelial dysfunction is associated with albuminuria and CKD in older adults. BMC Nephrol 2016;17:82.

38. Levey AS, Stevens LA, Schmid CH, Zhang YL, Castro AF, 3rd, Feldman $\mathrm{HI}$, et al. A new equation to estimate glomerular filtration rate. Ann Intern Med 2009;150:604-12.

39. Stevens LA, Schmid CH, Greene T, Zhang YL, Beck GJ, Froissart $M$, et al. Comparative performance of the CKD Epidemiology Collaboration (CKD-EPI) and the Modification of Diet in Renal Disease (MDRD) Study equations for estimating GFR levels above $60 \mathrm{~mL} / \mathrm{min} / 1.73 \mathrm{~m}^{2}$. Am J Kidney Dis 2010;56:486-95.

40. Florkowski CM, Chew-Harris JS. Methods of estimating GFR - different equations including CKD-EPI. Clin Biochem Rev 2011;32:75-9.

41. Ihling C, Szombathy T, Bohrmann B, Brockhaus M, Schaefer HE, Loeffler BM. Coexpression of endothelin-converting enzyme-1 and endothelin-1 in different stages of human atherosclerosis. Circulation 2001;104:864-9. 